\title{
Differences in self-reported weekend catch up sleep between children and adolescents with and without primary hypertension
}

\author{
Neena Gupta ${ }^{*}$ DD, Louise Maranda ${ }^{2}$ and Rakesh Gupta ${ }^{3}$
}

\begin{abstract}
Background: The data on the association of sleep duration and blood pressure in the pediatric age group have been mixed and most studies have focused on weekday sleep duration. The purpose of this study was to compare the weekday and weekend sleep patterns between children and adolescents with newly diagnosed primary hypertension and a normotensive control group.

Methods: Children and adolescents from a pediatric nephrology clinic, aged 6-18 years with newly diagnosed primary hypertension were compared to an age and sex matched normotensive control group from a general pediatric clinic. The questions about bed time and getting out of bed times from the Pediatric Sleep Questionnaire (PSQ) were used to obtain weekday and weekend bed time, getting out of bed time and sleep duration. The Pediatric Daytime Sleepiness Scale (PDSS) was used to assess subjective sleepiness.

Results: In both groups of 60 subjects each, weekday total sleep time was similar. Subjects in both groups went to bed later and woke up later on the weekends. However, in the hypertensive group, weekend getting out of the bed time was earlier (8:52 AM \pm 93 min vs. 9:36 AM $\pm 88 \mathrm{~min}, p=0.013$ ) and weekend catchup sleep was about 40 min less ( $62.8 \pm 85.5$ vs. $102.7 \pm 84.9, p=0.035)$. Hypertensive children perceived less subjective sleepiness (PDSS scores $8.28 \pm 4.88$ vs. $10.63 \pm 5.41, p=0.007)$. The $p$ values were calculated after adjusting for body mass index (BMI), race, daytime nap, caffeine use, sleep related breathing disorder (SRBD) scale and periodic limb movement of sleep (PLMS) scale subcomponents of the PSQ.

Conclusions: Hypertensive children obtained less weekend catch up sleep and reported less subjective sleepiness compared to the control group. More weekend sleep may potentially mitigate the effect of weekday sleep deprivation on blood pressure.
\end{abstract}

Keywords: Sleep duration, Blood pressure, Hypertension, Children, Adolescents

\section{Background}

It is important to understand the factors affecting blood pressure (BP) in children as elevated BP in childhood predicts BP in adulthood [1]. Childhood hypertension has been shown to cause target organ damage like left ventricular hypertrophy, increased carotid intimal thickness and microalbuminuria that are known to predict future cardiovascular events [2].

\footnotetext{
* Correspondence: neena.gupta@umassmemorial.org

${ }^{1}$ University of Massachusetts Children's Medical Center, Division of Pediatric

Nephrology, 55 Lake Avenue North, Benedict Bldg, A2 210, Worcester, MA 01655, USA

Full list of author information is available at the end of the article
}

Etiology of hypertension in pediatric population is multifactorial and has been attributed to genetic, renal, endocrine, excess weight, sedentary lifestyle and sleep related factors. Recent interest in the relationship between sleep and BP comes at a time when the selfreported sleep duration in adolescents has been declining over last 20 years [3] and remains significantly below the recommended amount of sleep [4].

Commonly proposed mechanisms for increase in BP due to insufficient sleep include sympathetic system over-activity, higher cortisol levels and endothelial dysfunction [5-7]. One night of sleep deprivation can also increase arterial stiffness [8]. Other metabolic 
effects of insufficient sleep like insulin resistance, increased inflammatory markers, and changes in appetite with a preference for salty and sweet foods may also impact blood pressure indirectly $[5,6]$. Most of these studies have assessed the effects of short term sleep deprivation only.

Data on the effect of habitual sleep duration on $\mathrm{BP}$ in pediatric age group are limited and they show mixed results [9]. Some studies show an association between shorter sleep duration and BP or hypertension status [10-13], while others have found no relationship or the relationship was not significant after adjusting for covariates [14, 15] or found mixed results for different age groups [16]. Still other studies have reported an association of long sleep duration with higher odds of high blood pressure in females [17].

Most of the pediatric studies evaluating the association between sleep duration and BP have been in a cohort of normal children, have used different measures of sleep duration, and different cut offs for "short sleep duration" [10-17]. Some studies have estimated the sleep duration by averaging self-reported sleep time [10] or actigraphic sleep duration [11] over 7 days, some have used $1 \mathrm{~d}$ actigraphic sleep duration on a school day [13], some have used the self-reported sleep duration on school days only [17], some have used the 3 to 5 day actigraphic sleep duration on school days only [14] and others have used self-reported sleep duration from a question about "usual sleep duration" without reference to any day of the week $[12,15,16]$. However, the typical sleep pattern in school children in the United States is that of less sleep on weekdays and catch up on weekends; primarily by waking up later [18]. The main driver for such behavior is the need of waking up early for school on weekdays in the face of a biologically delayed circadian sleep wake rhythm at this age. It is a common professional recommendation that teenagers should not sleep in on weekends [19] to minimize "jet lag" but there is very limited evaluation of the effect of sleeping in on weekends on BP. A survey of Korean middle aged adults showed that the weekend catchup sleep was associated with lower prevalence of hypertension [20]. These limited data suggest that the contribution of weekend catch up sleep is not fully captured by the sleep duration averaged over 7 days or measured only on weekdays as has been done in most studies.

In this study, we compared hypertensive children with a group of age and sex matched normotensive children with respect to weekday and weekend sleep parameters such as sleep duration, bedtime, out of bed time and weekend-weekday sleep difference. We hypothesized that the hypertensive group would obtain less catch up sleep on the weekends.

\section{Methods}

Subjects for this study were identified from a cohort of subjects recruited from the pediatric nephrology clinic and a control group from the general pediatric clinic between Jan 2013 to Jan 2016 to study the prevalence of sleep problems in the pediatric nephrology patients (Fig. 1). From the pediatric nephrology patients, a subset of 63 children diagnosed with new onset primary hypertension (HBP group) was the group of interest for this study of which 3 patients were excluded due to incomplete diagnostic work up. As age and sex have a large effect on the BP in children, an age (matched to $<3$ months difference) and sex matched control group of 60 normotensive children was identified from the general pediatric clinic for the robust control of these confounders. The study was approved by our Institutional Review Board.

Patients between the ages of 6-18 years were approached on the days when trained study personnel (nurse practitioner and medical students) were available. At the time of consenting, both, the guardian and the patient completed the sleep questionnaires together. Inclusion criteria required that the Body Mass Index (BMI) and clinic BP data be available in the medical record for both groups within 6 months. Exclusion criteria for both groups were: use of antihypertensive medications, any severe neurologic disorders (brain malformations, mental retardation, and cerebral palsy), or other severe co-morbidities (e.g. uncontrolled asthma, diabetes, chronic pain, symptomatic heart failure). For the HBP group, only children with newly diagnosed primary hypertension were included.

Demographics, BMI, BP and co-morbidities were extracted from the medical records. BMI categories were based on the age and sex specific percentiles defined by the US Centers for Disease Control and Prevention [21]. $\mathrm{BMI}<85$ th percentile was considered as normal weight, $\geq 85$ th percentile but $<95$ th percentile was considered as overweight and $\geq 95$ th percentile was considered as obese. BP was measured in the clinic using Welch Allyn Masimo SET Spot vital sign LXi instrument (model number 45MT0) by trained personnel. An average of all $\mathrm{BP}$ readings was taken when there were multiple BP readings available in one sitting in patients from the control group. In the HBP group, a physician repeated the BP measurement following the recommendations of the National High Blood Pressure Education Program Working Group on High Blood Pressure in Children and Adolescents to confirm HBP [22]. The patients were considered normotensive if systolic blood pressure (SBP) and/or diastolic blood pressure (DBP) was <90th percentile for age, sex and height, pre-hypertensive if SBP and/or DBP was $\geq 90$ th percentile but $<95$ th percentile and hypertensive if SBP and/or $\mathrm{DBP} \geq 95$ th percentile [22]. 


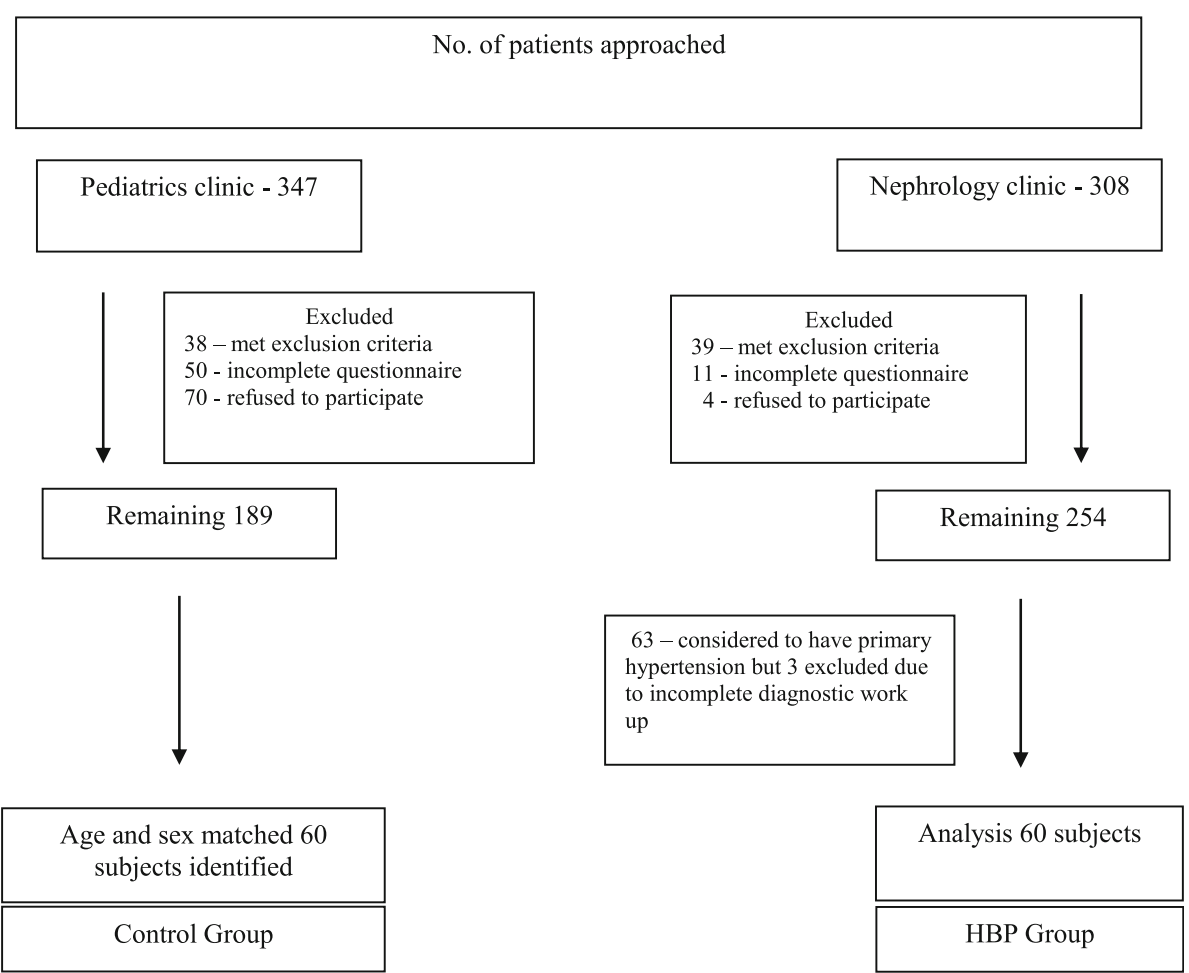

Fig. 1 Flow chart for patient selection

Questionnaires included the Pediatric Sleep Questionnaire (PSQ) [23] and the Pediatric Daytime Sleepiness Scale (PDSS) [24]. The PSQ is a validated instrument designed to assess various sleep symptoms. Sleep related breathing disorder (SRBD, most common form of SRBD being obstructive sleep apnea) and periodic leg movements during sleep (PLMS) scores were calculated using the SRBD [23] and PLMS [25] subscales of PSQ and a score of $>0.33$ was considered as an indicator of higher likelihood of the respective disorder [23, 25]. Weekday and weekend sleep duration was calculated from response to the following PSQ questions: "What time does your child usually go to bed during week (during school days)?", "what time does your child usually go to bed on weekend or vacation (when you are not in school)?", "what time does your child usually get out of bed on weekday mornings (during school days)?" and "what time does your child usually get out of bed on weekend or vacation mornings (when you are not in school)?" Weekend catch up sleep was calculated as the difference between weekend and weekday sleep duration. Bedtime and out of bed time were converted to decimal format for statistical analysis. The PDSS is a validated instrument for assessing daytime sleepiness in school age children with a score range of 0-32. A higher PDSS score indicates more sleepiness. Use of caffeinated drinks and daytime nap was identified (as yes or no) from the responses to the questions in the PSQ.

\section{Statistical analysis}

The final sample included 60 patients in each group (see Fig. 1). Continuous variables were compared between the two groups using independent sample t-tests and categorical variables were compared using chi-square tests. ANOVA was used to adjust for Body Mass IndexStandard Deviation Score (BMI-SDS), race, daytime nap, caffeine use, sleep related breathing disorder (SRBD) scale and Periodic limb movement in sleep (PLMS) scale subcomponents of PSQ. Linear regression and Pearson correlation coefficient ( $p$-value calculated for a unidirectional hypothesis predicting less sleep time to be associated with more sleepiness) were used to assess the relationship of the sleep duration to PDSS scores. IBM SPSS Statistical software package 24 was used for these calculations.

\section{Results}

\section{Subject characteristics}

Demographic and anthropometric data are presented in Table 1 . Of the 60 subjects in each group, $70 \%$ were boys and the mean age was $14.2 \pm 3.3$ years. As expected, BMI-SDS, SBP and DBP were significantly higher in the HBP group. Normal weight, overweight, and obesity were noted in 21 (35\%), $11(18.3 \%)$ and $28(46.6 \%)$ in the HBP group and 45 (75\%), 6 (10\%), and $9(15 \%)$ in the control group respectively. There were no significant differences between the two groups in the racial 
Table 1 Baseline and sleep parameters

\begin{tabular}{|c|c|c|c|c|}
\hline & $\begin{array}{l}\text { HBP group } \\
(\text { mean } \pm \text { SD or } \%)\end{array}$ & $\begin{array}{l}\text { Control group } \\
(\text { mean } \pm \text { SD or \%) }\end{array}$ & $P$ value & $\begin{array}{l}P \text { value adjusted for Race, } \\
\text { Caffeine use, BMI-SDS, Daytime } \\
\text { Nap, SRBD scale and PLMS scale }\end{array}$ \\
\hline $\mathrm{N}$ & 60 & 60 & & \\
\hline Age (years) & $14.2 \pm 3.3$ & $14.2 \pm 3.3$ & 0.96 & \\
\hline Gender (Male) & 70 & 70 & 0.70 & \\
\hline Race (whites) & 58 & 62 & 0.371 & \\
\hline BMI & $25.8 \pm 5.5$ & $21.9 \pm 4.2$ & $<0.00$ & \\
\hline BMI- SDS & $1.26 \pm 0.99$ & $0.55 \pm 0.85$ & $<0.00$ & \\
\hline BMI Percentile & $82 \pm 24$ & $66 \pm 23$ & $<0.00$ & \\
\hline SBP mm Hg & $131.5 \pm 13.8$ & $112.7 \pm 9.6$ & $<0.00$ & \\
\hline DBP mm Hg & $79.4 \pm 7.8$ & $71.3 \pm 6.7$ & $<0.00$ & \\
\hline SRBD $>0.33$ & $18(30)$ & $12(20)$ & 0.31 & \\
\hline PLMS $>0.33$ & $20(33.3)$ & $17(28.3)$ & 0.64 & \\
\hline $\begin{array}{l}\text { WD Bedtime (in decimal) } \\
\text { (hour:min) }\end{array}$ & $\begin{array}{l}9.70 \pm 1.02 \\
9: 42 \mathrm{PM}\end{array}$ & $\begin{array}{l}9.78 \pm 0.99 \\
9: 47 \mathrm{PM}\end{array}$ & 0.65 & 0.918 \\
\hline $\begin{array}{l}\text { WE Bedtime (in decimal) } \\
\text { (hour:min) }\end{array}$ & $\begin{array}{l}10.97 \pm 1.34 \\
10: 59 \mathrm{PM}\end{array}$ & $\begin{array}{l}11.25 \pm 1.40 \\
11: 15 \mathrm{PM}\end{array}$ & 0.28 & 0.209 \\
\hline $\begin{array}{l}\text { WD OOB time (in decimal) } \\
\text { (hour:min) }\end{array}$ & $\begin{array}{l}6.55 \pm 0.88 \\
6: 33 \mathrm{AM}\end{array}$ & $\begin{array}{l}6.47 \pm 64 \\
6: 28 \text { AM }\end{array}$ & 0.62 & 0.526 \\
\hline $\begin{array}{l}\text { WE OOB time (in decimal) } \\
\text { (hour:min) }\end{array}$ & $\begin{array}{l}8.86 \pm 1.55 \\
8: 52 \mathrm{AM}\end{array}$ & $\begin{array}{l}9.6 \pm 1.46 \\
9: 36 \mathrm{AM}\end{array}$ & 0.007 & 0.013 \\
\hline WD TST (minutes) & $530.9 \pm 74.9$ & $519.6 \pm 76.6$ & 0.42 & 0.530 \\
\hline WE TST (minutes) & $593.7 \pm 79.1$ & $622.3 \pm 80.9$ & 0.053 & 0.081 \\
\hline WE-WD TST (minutes) & $62.8 \pm 85.5$ & $102.7 \pm 84.9$ & 0.012 & 0.035 \\
\hline PDSS total & $8.28 \pm 4.88$ & $10.63 \pm 5.41$ & 0.014 & 0.007 \\
\hline Daytime nap - Yes & $15(25)$ & $7(11)$ & 0.060 & \\
\hline Caffeine users & $16(26.6)$ & $22(36.6)$ & 0.33 & \\
\hline
\end{tabular}

$B M I$ Body mass index, BMI-SDS body mass index standard deviation score, SBP systolic blood pressure, DBP diastolic blood pressure, SRBD sleep related breathing disorder scale score, PLMS periodic limb movements in sleep scale score, WD Bedtime weekday going to bed time, WE Bedtime weekend going to bed time, WD $O O B$ week day getting out of bed time, WE OOB weekend getting out of bed time, WD TST weekday total sleep time, WE TST weekend total sleep time, WE-WD TST Weekend catchup sleep time calculated as WE TST minus WD TST, PDSS pediatric daytime sleepiness scale

distribution, the proportion of the subjects taking caffeine, daytime nap, and abnormal SRBD and PLMS subscale scores from the PSQ. Racial composition of the HBP group was: white/not Hispanic 35 (58\%), Hispanic 15 (25\%), African-American 3 (5\%), Asian-American 2 (3\%), other $5(8 \%)$ and in the control group was: white/ not Hispanic 37 (62\%), Hispanic 10 (17\%), AfricanAmerican 6 (10\%), Asian-American 4 (7\%), other 3 (5\%).

\section{Sleep patterns}

See Table 1 for details and the parameters used to calculate adjusted $p$ value. In our local area, school hours are approximately from 7:10-8:15 AM to 1:45-3:00 PM. On weekdays, going to bed and getting out of the bed times were similar in both the groups resulting in similar mean weekday total sleep times (in minutes, 530.9 \pm 74.9 in the HBP group vs. $519.6 \pm 76.6$ in controls, $p=0.42$ and after adjustment 0.53 ). Weekend total sleep time tended to be longer in control subjects (in minutes, $622.3 \pm 80.9$ vs. $593.7 \pm 79.1, p=0.053$ and after adjustment 0.081 ). The weekend bedtime was not statistically different between the groups. Subjects in both groups went to bed later on weekends as compared to weekdays. Compared to weekdays, weekend getting out of bedtime was also later in both groups. However, subjects in the control group woke up later than the subjects in the HBP group on weekends (9:36 AM $\pm 88 \mathrm{~min}$ vs. 8:52 $\mathrm{AM} \pm 93 \mathrm{~min}, p=0.007$ and after adjustment 0.013). As a result, children in HBP group woke up $2 \mathrm{~h}$ and 19 min later on the weekend as compared to weekdays and had $62.8 \mathrm{~min}$ of weekend catchup sleep whereas those in the control group woke up $3 \mathrm{~h}$ and $8 \mathrm{~min}$ later on the weekend and had 102 . $7 \mathrm{~min}$ of weekend catchup sleep. Thus, the weekend catch up sleep in control group was about $40 \mathrm{~min}$ longer and significantly more than in the HBP group $(102.7 \pm 84.9$ vs. $62.8 \pm 85.5 \mathrm{~min}, p=0.012$ and after adjustment 0.035$)$. 


\section{Subjective sleepiness}

In both groups, subjective sleepiness as measured by PDSS was inversely correlated with the weekday sleep time (Fig. 2) and weekday sleep time was inversely correlated with the weekend catch up sleep time (Fig. 3). This suggests that in both groups, children getting less sleep on the weekdays feel sleepier and children who feel sleepier obtained more catch up sleep on the weekend. However, when comparing the two groups, subjects in HBP group perceived significantly less subjective sleepiness (PDSS scores $8.28 \pm 4.88$ vs. $10.63 \pm 5.41$, $p=0.014$ and after adjustment 0.007 ) even though there was no significant difference in the weekday sleep time between the two groups (Table 1). The slope for the increase in sleepiness and resultant catch up sleep in response to weekday sleep duration was same in both groups but the magnitude of this response was lower in HBP group (the regression lines in the HBP group were lower in magnitude but parallel to control group, Figs. 2 and 3).

\section{Discussion}

In this study, we compared the sleep parameters (sleep duration, sleep timing and subjective daytime sleepiness) in a group of newly diagnosed hypertensive children and adolescents with an age and sex matched normotensive control group. While the sleep parameters on weekdays were similar between the groups, major findings from our study are that the subjects in the HBP group woke up earlier on weekends and obtained less catch up sleep on weekends compared to the normotensive control group. The HBP group also reported less subjective sleepiness compared to the control group. These significant differences in the sleep parameters persisted after adjustments (for BMI-SDS, race, daytime nap, caffeine use, SRBD scale and PLMS scale) in our study suggesting an independent association of weekend catch up sleep and BP.

Previous studies in children and adolescents looking at the association of sleep duration on BP have produced inconsistent results. Au et al. and Mezick et al. reported an association between shorter sleep (average of 7 days) and higher BP $[10,11]$. On the other hand, Paciencia et al. found that the odds of high BP (> 90th percentile) were increased in female adolescents sleeping $>9.5 \mathrm{~h}$ per night (measured on week days only) compared to those sleeping < $8.5 \mathrm{~h}$ nightly [17]. Javaheri et al. examined sleep duration on school days and found short sleep duration to be associated with higher odds of prehypertension but the association was not significant after adjustment for sex, BMI and socioeconomic status [14]. Peach et al. showed that in 6th grade children, schoolnight sleep duration, weekend sleep duration and daytime sleepiness directly predicted BMI and indirectly predicted risk for hypertension via association with BMI [26]. Meininger et al. also found an inverse relationship between sleep duration and BP by measuring sleep duration and Ambulatory Blood Pressure Monitor (ABPM) together on a school day [13]. The role of weekend catch up sleep was not captured in any of these studies due to their different study designs.

Our study differs from these studies as we assessed weekday and weekend sleep separately. In contrast to other studies that have looked at the association between sleep duration and blood pressure in a single cohort, we compared sleep parameters of a newly diagnosed hypertensive group to an age and sex matched normotensive group. As all school going children are forced to conform to similar schedules on weekdays; it is understandable that the weekday bed time, getting out of bed time and total sleep time were similar in both the HBP and the normotensive groups. Based on the "2006 sleep in

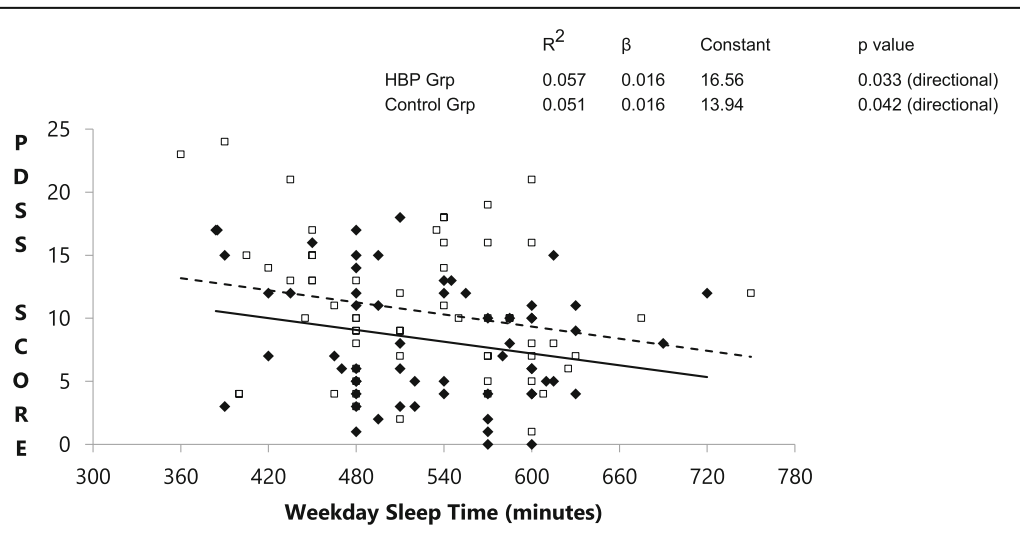

Fig. 2 Relationship between weekday sleep time and subjective sleepiness. Weekday sleep time shows an inverse relationship with subjective sleepiness (PDSS score - pediatric daytime sleepiness scale) in both groups. The parallel but lower regression line in the HBP group (High blood pressure group) suggests qualitatively similar but quantitatively lower response to weekday sleep deprivation. (HBP group - solid diamonds, solid line; Control group - open squares, dashed line) 


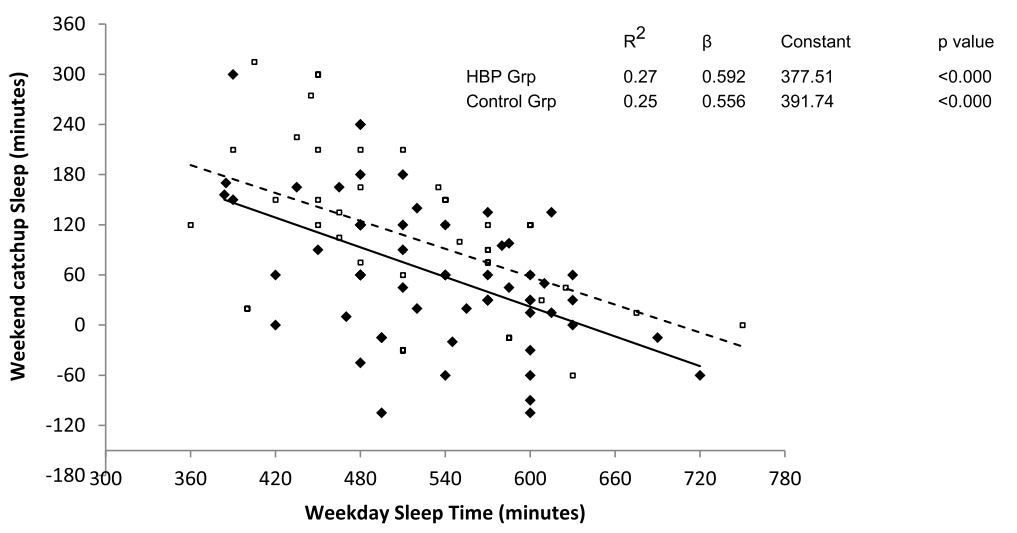

Fig. 3 Relationship between weekday sleep time and weekend catchup sleep time. Weekday sleep time shows an inverse relationship with weekend catchup sleep time in both groups. The almost parallel but lower regression line in the HBP group (High blood pressure group) suggests qualitatively similar but quantitatively lower response. Many more children in HBP group have a negative weekend catch up sleep time. (HBP group - solid diamonds, solid line; Control group - open squares, dashed line)

America Poll", self-reported weekday bed time and out of bedtime for 7th, 8th and 9th graders were 2152, 2153, 2215 and $0635,0636,0628 \mathrm{~h}$ respectively and weekend bed time and out of bed time were 2305, 2326, 2353 and 0912, 0921, 0954 h respectively [18]. In our study which also used self-reported questionnaire data, the overall timings are very similar to the national data. The weekday bed time and out of bedtime means were 2142 and $0633 \mathrm{~h}$ for HBP group and 2147 and $0628 \mathrm{~h}$ for control group respectively. Weekend bed time and out of bed time were 2259 and $0852 \mathrm{~h}$ in HBP group and 2315 and $0936 \mathrm{~h}$ for control group respectively. The weekend out of bed time in control group was closer to the national average but it was earlier in HBP group. There was a mean difference of $44 \mathrm{~min}$ in our study in weekend out of bed time between HBP and control group.

There is a circadian phase delay with maturation of the biological regulation of sleep in adolescents which is responsible for their normal tendency to go to bed late and get out of bed late [18]. Besides this, psychosocial factors like social networking, pressure to be a high achiever, availability and use of new technology, also contribute to delay in going to sleep time although the need for total sleep time remains constant [18]. Wake up time could also be determined by external forces, like early school start times, to watch favorite television shows or athletic commitments. Students tend to wake up late on weekends due to both phase delay and to catch up on sleep debt accumulated on weekdays [18]. It is likely that the sleep pattern on weekend has better circadian alignment to their natural underlying rhythm whereas on weekdays, the sleep pattern is less aligned with their natural circadian rhythm. Students who wake up earlier on weekend may not get the full benefit of this week end catch up sleep. There are limited number of studies looking at the relationship between weekend catch up sleep and BP. A Korean survey of middle aged adults showed that more weekend catch up sleep was associated with lower risk of prevalent hypertension [20]. Studying this association in children may be more relevant to understanding the relationship between weekend catch up sleep and blood pressure because in adult studies, sleep duration is measured many years after the development of hypertension. Race has also been suggested as a factor affecting sleep duration and bedtimes [27]. Minorities comprised 40\% of our subjects. The differences in sleep parameters between hypertensive and normotensive subjects remained significant after adjustment for race.

In this study, the control group reported more subjective sleepiness even though they had same weekday sleep time and longer weekend sleep time as compared to the HBP group. The weekday sleep duration was inversely correlated to subjective sleepiness in both groups confirming that both groups respond to sleep loss qualitatively in the same way but children in the hypertensive group seem to have lower magnitude of subjective sleepiness (Figs. 2 and 3). Also, weekday total sleep time was inversely proportional to the weekend catch up sleep in both groups but HBP group had comparatively less weekend catch up sleep. Several explanations are possible for this pattern. It is possible that some individuals have learned behaviorally to ignore or become desensitized to the sensation of sleepiness due to competing priorities. In our clinical practice, we have observed that some children wake up on weekend in time to watch their favorite television shows. It is also known that the ability to feel sleepiness (subjective sleepiness) is an intrinsic biological trait [28] and individuals have differential vulnerability to sleep loss [29]. Higher level of subjective sleepiness in the control group may either allow or force them to obtain more catch-up 
sleep on weekends. The dissociation between subjective sleepiness and physiological impairment due to insufficient sleep has been confirmed for some neurological parameters. Cognitive impairment $[30,31]$ and abnormalities on fMRI (functional Magnetic Resonance Imaging) of the brain [32] have been demonstrated even in short sleepers who may not report feeling sleepy.

Our study has several strengths. We have assessed weekday and weekend sleep duration separately. Our age and sex matched controls allowed for a more robust control of these confounders as these factors have a very large impact on BP in children and adolescents. We also confirmed the diagnosis of hypertension by ambulatory BP measurement. We compared sleep parameters of a group of children with high BP to a control group whereas, in other studies, the "association or relationship" of sleep duration to BP was evaluated in a single cohort of subjects. Confounding from sleep disorders such as obstructive sleep apnea or PLMS was addressed by adjusting for the SRBD and PLMS scale scores [23, 25].

At the same time, our study has some limitations. Subjects were recruited on days when study personnel were available. Although it was not a random sample, we do not suspect any systematic bias in the patient recruitment. As self-reported sleep time was calculated from response to questions, our data could be described as "nocturnal rest time" rather than "sleep time". Thus the actual sleep time is likely to be less than the calculated sleep time in our subjects. The large magnitude of weekend catch up sleep in both groups is an indication of insufficient sleep duration on weekdays. Other potential confounders such as physical activity level, extracurricular activities, socioeconomic status and substance use were not evaluated. Circadian rhythm markers of individual participants were not assessed but studies have shown that most children have a phase delay as they mature [18].

\section{Conclusions}

In conclusion, our data suggests that children with newly diagnosed primary hypertension obtain less weekend catch-up sleep by waking up earlier on weekend. Hypertensive children have more blunted "subjective sleepiness" response to insufficient sleep. Future studies evaluating the relationship between sleep and BP should consider the effect of weekend sleep in addition to weekday sleep. Additional information will help in counseling our patients regarding weekend sleep patterns.

\section{Abbreviations}

BMI-SDS: Body Mass Index-Standard Deviation Score; BP: Blood pressure; DBP: Diastolic blood pressure; HBP: High blood pressure; PDSS: Pediatric Daytime Sleepiness Scale; PLMS: Periodic leg movements of sleep; PSQ: Pediatric Sleep Questionnaire; SBP: Systolic blood pressure; SRBD: Sleep related breathing disorder

\section{Acknowledgements}

We would like to thank Eric Olson, MD for reviewing and critiquing the manuscript. We also thank Jennifer Kremer $^{2}$, CPNP, Meghan Gibson², MD and Jessica $\mathrm{Ngo}^{2}, \mathrm{MD}$ for help in recruiting subjects for this study.

${ }^{1}$ Mayo Clinic Center for Sleep Medicine, Rochester, MN

2 University of Massachusetts Children's Medical center, Worcester, MA

\section{Funding}

This study is supported by University of Massachusetts Medical School internal grant \# 109904.

\section{Availability of data and materials}

The datasets used for the current study are available from the corresponding author on reasonable request.

\section{Authors' contributions}

NG collected the data and drafted the manuscript. LM performed the statistical analysis. RG performed the statistical analysis and drafted the manuscript. All authors read and approved the final manuscript.

\section{Ethics approval and consent to participate}

Study approved by IRB at University of Massachusetts Children's Medical center, Worcester, MA. IRB number - 14556. Parents/Guardians provided consent, subjects provided verbal assent.

Consent for publication

Not applicable.

\section{Competing interests}

The authors declare that they have no competing interests.

\section{Publisher's Note}

Springer Nature remains neutral with regard to jurisdictional claims in published maps and institutional affiliations.

\section{Author details}

${ }^{1}$ University of Massachusetts Children's Medical Center, Division of Pediatric Nephrology, 55 Lake Avenue North, Benedict Bldg, A2 210, Worcester, MA 01655, USA. 'University of Massachusetts Memorial Medical Center,

Quantitative Health Sciences, Worcester, MA 01655, USA. ${ }^{3}$ PrimaCare Sleep

Center, Somerset, MA 02726, USA.

Received: 18 January 2018 Accepted: 28 March 2018

Published online: 05 April 2018

\section{References}

1. Chen X, Wang Y. Tracking of blood pressure from childhood to adulthood: a systematic review and meta-regression analysis. Circulation. 2008:117:3171-80.

2. Rao G. Diagnosis, epidemiology, and management of hypertension in children. Pediatrics. 2016;138:e20153616. https://doi.org/10.1542/peds.2015-3616.

3. Keyes KM, Maslowsky J, Hamilton A, Schulenberg J. The great sleep recession: changes in sleep duration among US adolescents, 1991-2012. Pediatrics. 2015:135:460-8.

4. Paruthi S, Brooks LJ, D'Ambrosio C, Hall WA, Kotagal S, Lloyd RM, Malow BA, Maski K, Nichols C, Quan SF, Rosen CL, Troester MM, Wise MS. Recommended amount of sleep for pediatric populations: a consensus statement of the American Academy of Sleep Medicine. J Clin Sleep Med. 2016;12:785-6.

5. Gangwisch JE. A review of evidence for the link between sleep duration and hypertension. Am J Hypertens. 2014;27:1235-42.

6. Mullington JM, Haack M, Toth M, Serrador JM, Meier-Ewert HK. Cardiovascular, inflammatory, and metabolic consequences of sleep deprivation. Prog Cardiovasc Dis. 2009;51:294-302.

7. Sauvet F, Leftheriotis G, Gomez-Merino D, Langrume C, Drogou C, Van Beers $P$, Bourrilhon C, Florence $G$, Chennaoui M. Effect of acute sleep deprivation on vascular function in healthy subjects. J Appl Physiol. 2010;108:68-75.

8. Sunbul M, Kanar BG, Durmus E, Kivrak T, Sari I. Acute sleep deprivation is associated with increased arterial stiffness in healthy young adults. Sleep Breath. 2014;18:215-20.

9. Matthews KA, Pantesco EJ. Sleep characteristics and cardiovascular risk in children and adolescents: an enumerative review. Sleep Med. 2016;18:36-49. 
10. Au CT, Ho CK, Wing YK, Lam HS, Li AM. Acute and chronic effects of sleep duration on blood pressure. Pediatrics. 2014;133:e64-72. https://doi.org/10 1542/peds.2013-1379.

11. Mezick EJ, Hall M, Matthews KA. Sleep duration and ambulatory blood pressure in black and white adolescents. Hypertension. 2012;59:747-52.

12. Kuciene R, Dulskiene V. Associations of short sleep duration with prehypertension and hypertension among Lithuanian children and adolescents: a cross-sectional study. BMC Public Health. 2014;14:255

13. Meininger JC, Gallagher MR, Eissa MA, Nguyen TQ, Chan W. Sleep duration and its association with ambulatory blood pressure in a school-based, diverse sample of adolescents. Am J Hypertens. 2014;27:948-55.

14. Javaheri S, Storfer-Isser A, Rosen CL, Redline S. Sleep quality and elevated blood pressure in adolescents. Circulation. 2008;118:1034-40.

15. Bayer $\mathrm{O}$, Neuhauser $\mathrm{H}$, von Kries $\mathrm{R}$. Sleep duration and blood pressure in children: a cross-sectional study. J Hypertens. 2009:27:1789-93.

16. Guo X, Zheng L, Li Y, Yu S, Liu S, Zhou X, Zhang X, Sun Z, Wang R, Sun Y. Association between sleep duration and hypertension among Chinese children and adolescents. Clin Cardiol. 2011;34:774-81.

17. Paciência I, Barros H, Araújo J, Ramos E. Association between sleep duration and blood pressure in adolescents. Hypertens Res. 2013;36:747-52.

18. Carskadon MA. Sleep in adolescents: the perfect storm. Pediatr Clin N Am. 2011;58:637-47.

19. Dennis Rosen, M.D. Why "sleeping in" on weekends isn't good for teens. Harvard Health Blog. [Online] January 11, 2013. http://www.health.harvard. edu/blog/why-sleeping-in-on-weekends-isnt-good-for-teens-201301115763. Accessed: 9 Apr 2017

20. Hwangbo Y, Kim WJ, Chu MK, Yun CH, Yang Kl. Association between weekend catch-up sleep duration and hypertension in Korean adults. Sleep Med. 2013;14:549-54.

21. Kuczmarski RJ, Ogden CL, Guo SS, Grummer-Strawn LM, Flegal KM, Mei Z, Wei R, Curtin LR, Roche AF, Johnson CL. 2000 CDC Growth Charts for the United States: methods and development. Vital Health Stat 11. 2002;246:1-190.

22. National High Blood Pressure Education Program Working Group on High Blood Pressure in Children and Adolescents. The fourth report on the diagnosis, evaluation, and treatment of high blood pressure in children and adolescents. Pediatrics. 2004;114(Suppl 2):555-76.

23. Chervin RD, Hedger K, Dillon JE, Pituch KJ. Pediatric sleep questionnaire (PSQ): validity and reliability of scales for sleep-disordered breathing, snoring, sleepiness, and behavioral problems. Sleep Med. 2000;1:21-32.

24. Drake C, Nickel C, Burduvali E, Roth T, Jefferson C, Pietro B. The pediatric daytime sleepiness scale (PDSS): sleep habits and school outcomes in middle-school children. Sleep. 2003;26:455-8.

25. Chervin RD, Hedger KM. Clinical prediction of periodic leg movements during sleep in children. Sleep Med. 2001;2:501-10

26. Peach H, Gaultney JF, Reeve CL. Sleep characteristics, body mass index, and risk for hypertension in young adolescents. J Youth Adolesc. 2015;44:271-84.

27. Combs D, Goodwin JL, Quan SF, Morgan WJ, Parthasarathy S. Longitudinal differences in sleep duration in Hispanic and Caucasian children. Sleep Med. 2016;18:61-6.

28. Mairesse O, De Valck E, Quanten S, Neu D, Cortoos A, Pattyn N, Theuns P, Cluydts R, Hofmans J. Sleepiness phenomics: modeling individual differences in subjective sleepiness profiles. Int J Psychophysiol. 2014;93:150-61.

29. Van Dongen HP, Vitellaro KM, Dinges DF. Individual differences in adult human sleep and wakefulness: leitmotif for a research agenda. Sleep. 2005; 28:479-96.

30. Frey DJ, Badia P, Wright KP Jr. Inter- and intra-individual variability in performance near the circadian nadir during sleep deprivation. J Sleep Res. 2004;13:305-15.

31. Van Dongen HP, Maislin G, Mullington JM, Dinges DF. The cumulative cost of additional wakefulness: dose-response effects on neurobehavioral functions and sleep physiology from chronic sleep restriction and total sleep deprivation. Sleep. 2003;26:117-26.

32. Curtis BJ, Williams PG, Jones CR, Anderson JS. Sleep duration and resting fMRI functional connectivity: examination of short sleepers with and without perceived daytime dysfunction. Brain Behav. 2016;12:e00576.

\section{Submit your next manuscript to BioMed Central and we will help you at every step:}

- We accept pre-submission inquiries

- Our selector tool helps you to find the most relevant journal

- We provide round the clock customer support

- Convenient online submission

- Thorough peer review

- Inclusion in PubMed and all major indexing services

- Maximum visibility for your research

Submit your manuscript at www.biomedcentral.com/submit
Biomed Central 